\title{
Adding Value to Continuous Measurement Data in Wells by Reversing the Thermal Profile of Reheating in Oil Reservoirs
}

Victor Costa da Silva, Paulo Couto, Franciane Conceição Peters

Copyright 2019, SBGf - Sociedade Brasileira de Geofísica

This paper was prepared for presentation during the $16^{\text {th }}$ International Congress of the Brazilian Geophysical Society held in Rio de Janeiro, Brazil, 19-22 August 2019.

Contents of this paper were reviewed by the Technical Committee of the $16^{\text {th }}$ International Congress of the Brazilian Geophysical Society and do not necessarily represent any position of the SBGf, its officers or members. Electronic reproduction or storage of any part of this paper for commercial purposes without the written consent of the Brazilian Geophysical Society is prohibited.

\section{Abstract}

The authors main objective in this work is to present, from different real conditions of the PPSBS (Santos Basin PreSalt Pole) wells, under various operating conditions, and through complex numerical models and optimization tools and problems conversely as the temperature signatures can be considered for the injectable effect, while the active zone, as well as the saturation of the rest environments as well as the cooling of the same. This objective will be reached though the solution of a inverse problem, regarding linear optimization aiming the history matching process. The optimization will gives the pursued information, critical for the reservoir management.

\section{Introduction}

The reservoir management process aims, among others, to act in a reactive or, preferably, proactive way to undesired events that impact oil production. As an example, it is possible to mention the increase of the water-oil and / or gas-oil ratio in the producing wells, due to the premature arrival of the injected fluids in the producing wells. Perhaps the greatest difficulty in this action is the traceability of the paths percolated by the injected fluids, both in the vicinity of the producing wells and the wells injectors.

In wells equipped with Selective Completion, the pressure downhole gauges (PDG) recorders, through their temperature data, represent an important tool to track the modification of saturations in each section evaluated. The objective of this work is to study the phenomena that are presented, using complex numerical models and inverse problem solving tools, to identify how some heating signatures may indicate the amount of injected fluid passed through each zone effectively, either by changing the saturation of the well environments or by cooling the well.

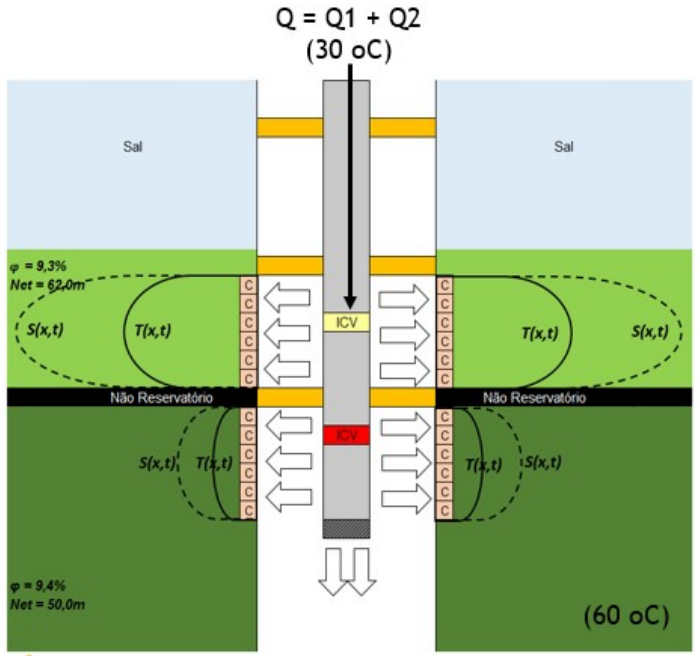

Figure 1: Typical configuration of a selection completion well injecting a rate $Q$ that is divided through the 2 intervals (Q1 and Q2)

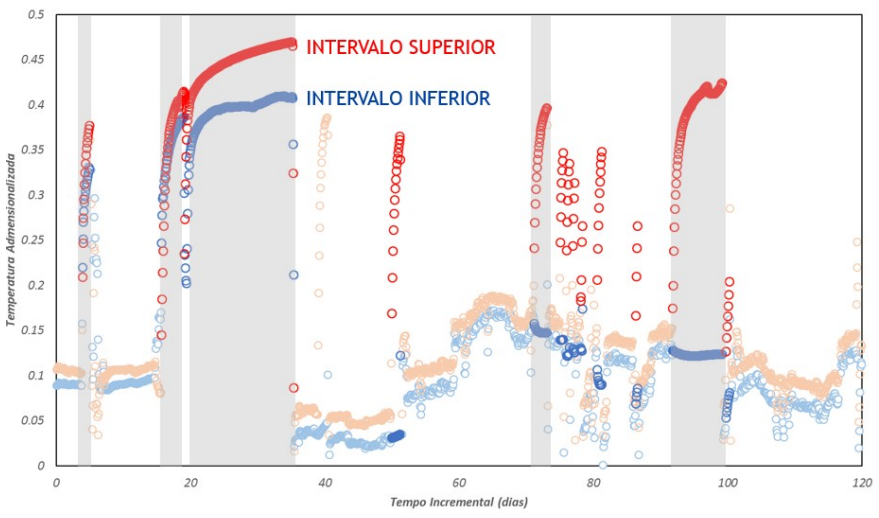

Figure 2: Normalized reheating data gathered from a Brazilian pre-salt well, showing the behavior of the temperature in two intervals after different injection periods

This sort of data can provide very useful information regarding fluid distribution alongside the reservoir, allowing the reservoir professionals to anticipate unexpected scenarios of injection and, therefore, avoids premature gas and water breakthrough.

\section{Method}

The simulations to estimate the region perturbed by the fluid injected from the temperature data in the wells are carried out in a static regime and are given through numerical modeling coupled between the Hydraulic Diffusivity Equation (EDH) and the Energy Balance 
Equation (EBE). The latter, in turn, will have all its main terms considered, among them the thermal conductivity, advection, compression and viscous dissipation. Moreover, since the evaluation must be done at separate and non-thermally isolated intervals, it is also necessary to couple the numerical solutions of the adjacent two layers. The numerical model considers a one-dimensional cylindrical grid, making it possible to adjust the measured data to define the volume effectively injected in each interest interval. Such a process of inversion of the temperature data will be performed from non-linear optimization methods and the parameters will be the spatial variation of the physical properties, such as the initial temperature field itself and the saturation field.

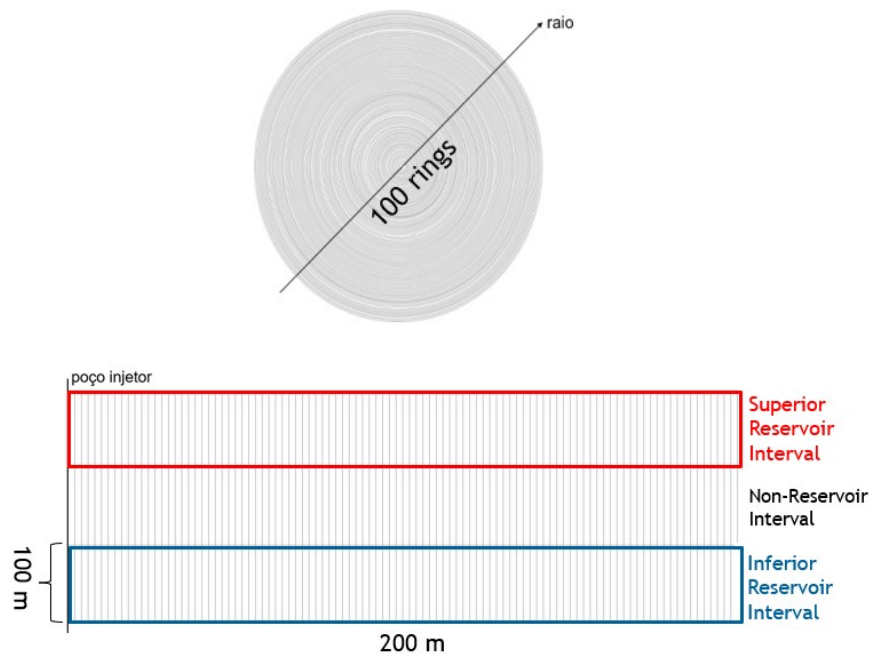

Figure 3: Unidimensional cylindrical grid model used in this work, composed by 2 coupled layers

For developing the embedded simulations mentioned above it is necessary to define several properties, such as hydraulical, thermal, and the initial temperature, pressure and saturation fields. Therefore the entire workflow is developed according to bellow, and finally the development in time of the temperature field is given through the EBE and HDE embedded solution.

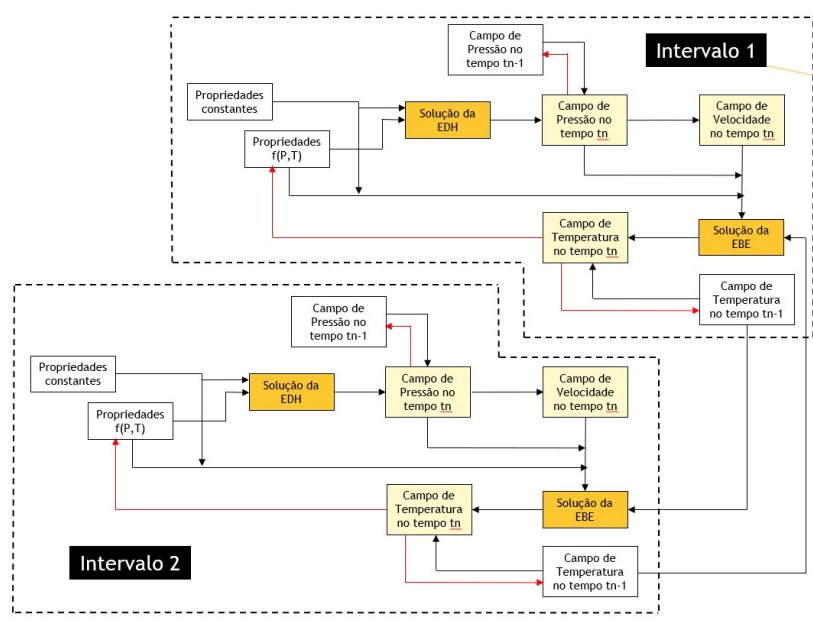

Figure 4: Workflow to reach the numerical solution of the physical problem described

Finally, with this outcome, it is possible to start the inverse problem solution, where a linear optimization with provide the ideal temperature and saturation initial fields in both intervals the matches the hard data obtained form the PDGs. The linear optimization might be given from parametrization of the fields or optimizing cell by cell of the grid.

\section{Results}

Through the mathematical modelling described before if is possible to assess the evolution of field temperature with time. Following the behavior of temperature in the well position it is possible to compare with the hard data and, eventually, match the history previously obtained.

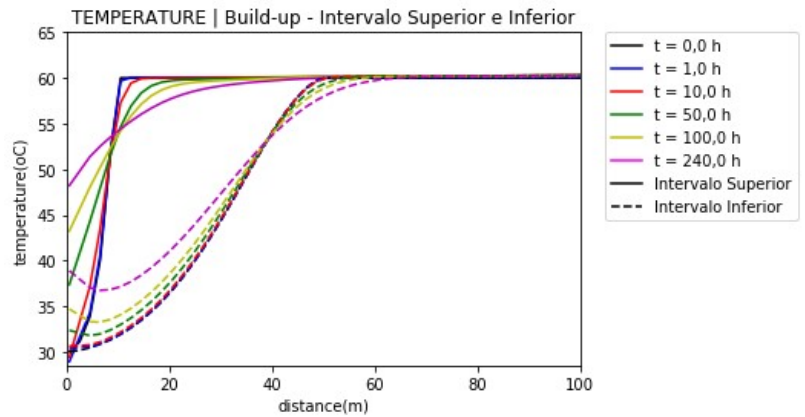

Figure 5: Behavior of the temperature along the reservoir model in the 2 layers and in different period of time

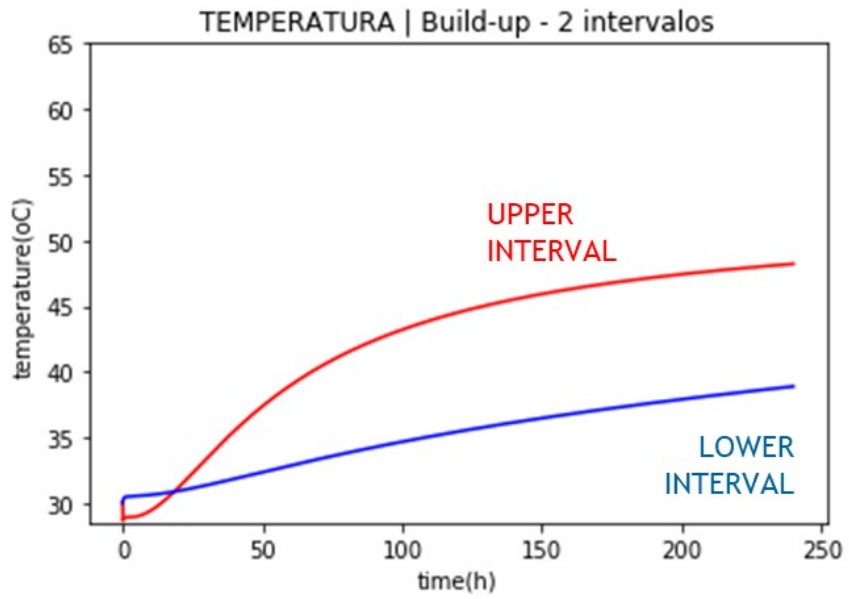

Figure 6: Behavior of the temperature across time in the well position for the 2 intervals

Furthermore this work presents sensibility analysis on how the different parameters of the full solution modify the final outcome. Amongst these parameters one can mention the thermal properties, like thermal conductivity, specific gravity, thermal capacity, etc; hydralical parameters like porosity and permeability, fluid viscosity, etc. But the most relevant parameters in the final solution 
is the temperature and saturation initial condition. These parameters will be the target of the inverse problem.

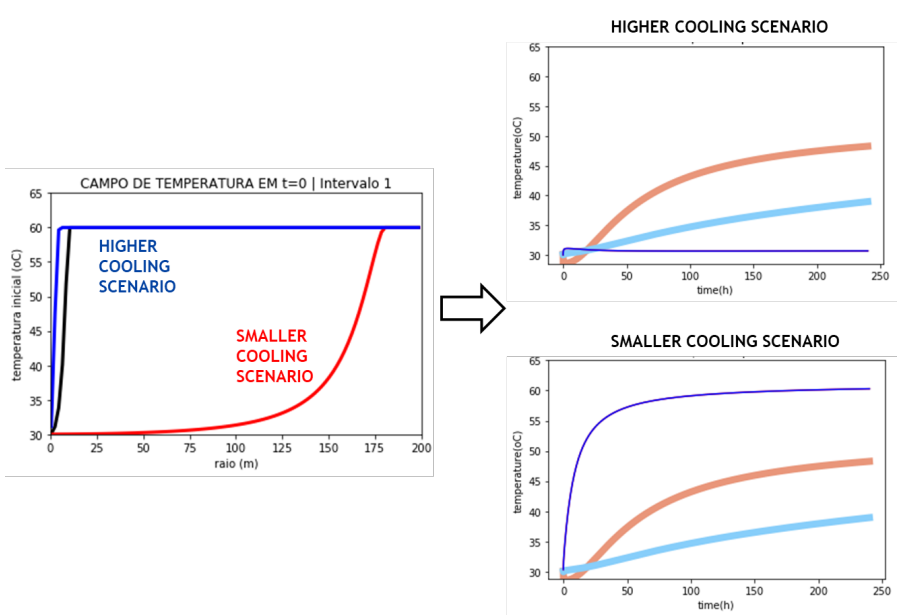

Figure 7: Sensibility of the temperature field with 2 different initial temperature conditions. In a higher cooling scenario the numerical solution gives a very low reheating profile. In a smaller cooling scenario on sees a very fast reheating scenario.

The following plots presents an example of the inverse problem that this work intends to solve, where a optimized initial temperature and saturation field leads the numerical to a very accurate matching with the hard data and, hence, the eventual conclusions.

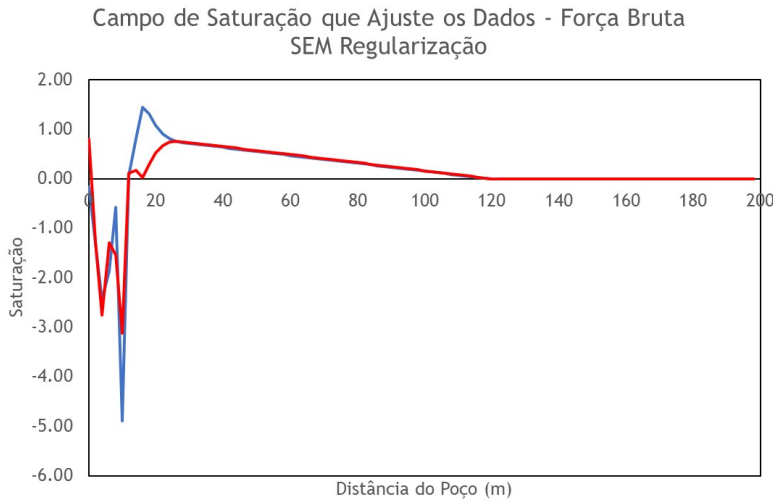

Campo de Temperatura que Ajuste os Dados - Força Bruta SEM Regularização

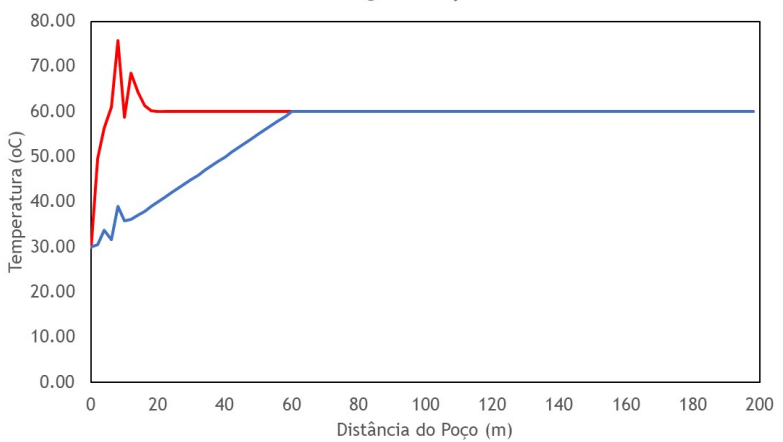

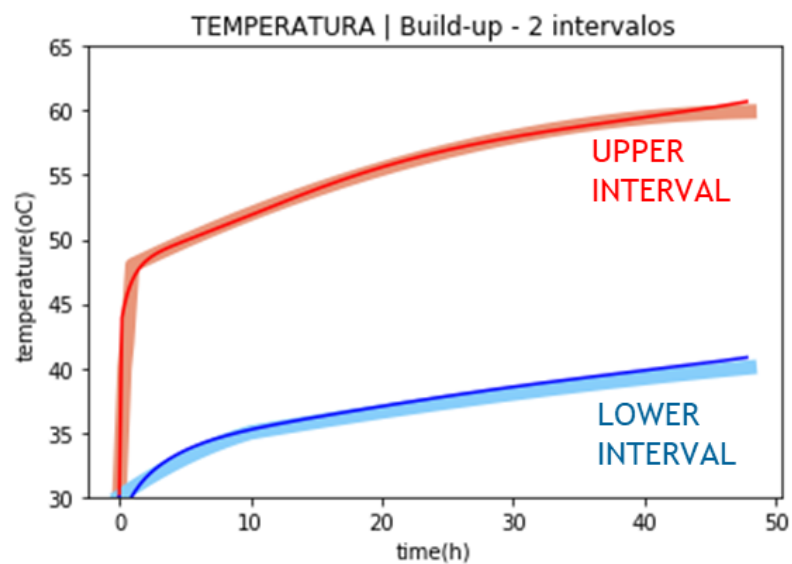

Figure 8: Hard data matched through the numerical model developed in the work and a linear optimization process trying to optimize the initial temperature and saturation cell by cell. However on can notice that the linear optimization generates unphysical values for temperature and saturation. This might be corrected in the parameterization process.

\section{Conclusions}

The results of this work can provide an important tool to assist in the management of oil reservoirs, denning one of the biggest uncertainties in the management of wells operating in more than one interval, which is the determination of the flow rates effectively injected into each of the interest intervals . In addition, it will allow a better understanding of the phenomenon that is observed recurrently in the injector wells of the Brazilian pre-salt, which is the discrepancy between the heating profile in the intervals of the injector wells. Finally, it will also allow for a more profound discussion on the value of EBE terms on the bottom line.

\section{Acknowledgments}

I acknowledge Petrobras and COPPE for given me the opportunity of developing such work, as well as the resources necessary.

I also acknowledge professors Paulo Couto and Fraciane Peters for the support in this journey. 


\section{References}

INCROPERA, F. P., DEWITT, D. P. Transferência de

Calor e Massa, Sétima Edição, 2014

OZISIK, M/ N. Boundary Value Problems of Heat

Conduction, Segunda Edição, 2002.

DAKE, L. P. The Practice of Reservoir Engineering,

Edição Revisada, 2008

DAKE, L. P. The Practice of Reservoir Engineering, Edição Revisada, 2008

SILVA. V. C, GLITZ, K. L. Z. How to Improve the Use of PDG Data on Injection Wells: A Temperature Build-Up

Approach, OTC 2017 